\title{
El síndrome metabólico en adultos, en el Perú
}

\author{
Jaime Pajuelo 1,2, José Sánchez 3,4
}

Resumen

Palabras clave
Objetivo: Conocer la prevalencia del síndrome metabólico en la población adulta del Perú. Materiales y Métodos: Se estudió 4091 personas mayores de 20 años. El50,4\% correspondióal género femenino y 49,6\% al masculino. Estas personas fueron elegidas sobre la base de un muestreo por conglomerado trietápico, que representa el nivel nacional y los siguientes ámbitos: Lima metropolitana, resto de la costa, sierra urbana, sierra rural y selva. A todos se les tomó el peso, la talla, la circunferencia de la cintura, la presión arterial, y se les dosó triglicéridos, colesterol HDL y glucosa. Para el diagnóstico del síndrome metabólico se utilizó el criterio del National Cholesterol Education Program ATP III (Adult Treatment Panel). Resultados: La prevalencia nacional del síndrome metabólico fue 16,8\%. Lima metropolitana (20,7\%) yel resto de la costa $(21,5 \%)$ fueron los únicos ámbitos que estuvieron por encima de la prevalencia nacional. La sierra rural es la que presentó los valores más bajos, con 11,1\%. El género femenino (26,4\%) superó ampliamente al masculino (7,2\%). El síndrome metabólico fue más prevalente en las personas con obesidad que en las que tenían sobrepeso. A mayor edad, mayor presencia del síndrome metabólico. Conforme se incrementó la circunferencia de la cintura, las otras variables lo hicieron de la misma manera. Conclusiones: En el país, 2680000 personas presentaron el síndrome metabólico, lo que significa que una gran cantidad de personas se encuentra en riesgo de su salud por las diversas alteraciones que le pueden ocurrir. Conociendo que la principal causa de este problema es el sobrepeso y la obesidad, hay que realizar estrategias que permitan combatir lo mencionado. Estas estrategias son ampliamente conocidas: tener una alimentación saludable y realizar una actividad física.

Metabolismo; obesidad; trastornos de ingestión de alimentos; hábitos alimenticios; ejercicio.

\begin{abstract}
Adult metabolic syndrome in Peru
Abstract

Objective: To determine the prevalence of metabolic syndrome in Peruvian adult population. Materials and Methods: We studied 4091 persons over 20 year-old; 50,4\% belonged to the female gender and 49,6\% to the male gender. These persons were chosen by a triepatic conglomerate sample which represents national level and the following spaces: Metropolitan Lima, remaining coast, urban highland, rural highland, and the selva. Weight, height, waist circumference, arterial blood pressure were determined as well as triglycerides, HDL cholesterol and glucose. For metabolic syndrome diagnosis the ATP III criteria were used. Results: National prevalence of metabolic syndrome was 16,8\%. In Metropolitan Lima (20,7\%) and the remaining coast (21,5\%) the metabolic syndrome was above national prevalence. The lower
\end{abstract}

1 Servicio de Endocrinología. Hospital Nacional Dos de Mayo. Lima, Perú.

2 Instituto de Investigaciones Clínicas. Facultad de Medicina, UNMSM. Lima, Perú.

3 Director Ejecutivo de VigilanciaAlimentario Nutricional. Centro Nacional de Alimentación y Nutrición (CENAN). Lima, Perú.

4 Instituto Nacional de Salud (INS). Ministerio de Salud. Lima, Perú. values were found in rural highland with $11,2 \%$. Female gender (26,4\%) broadly exceeded the male gender (7,2\%). Metabolic syndrome was more prevalent in obese rather than overweight persons. Metabolic syndrome increased as one gotolder. As weight circumference increased, the other variables followed the same pattern. Conclusions: In Peru 280000 persons presentmetabolic syndrome and are in great health risk, because of the different alterations which may occur. As the main reason of this problem is overweight and obesity, it is necessary to implement strategies to fight them. These strategies are widely known: healthy nourishment and physical activity.

Key words: Metabolism; obesity; eating disorders; food habits; exercise.

\section{INTRODUCCIÓN}

El síndrome metabólico (SM) ha sido reconocido hace más de 80 años y ha recibido diversas denominaciones a través del tiempo. De ninguna manera se trata de una única enfermedad, sino fundamentalmente 
de una asociación de problemas que por sí solos generan un riesgo para la salud y que en su conjunto se potencializan; o simplemente, una relación de factores que se relacionan estadísticamente $\left({ }^{1}\right)$.

La causa de estos problemas está dada por la combinación de factores genéticos y socioambientales relacionados a los cambios en los estilos de vida, especialmente la sobrealimentación y la inactividad física. Sin embargo, hay que considerar que algunos individuos están genéticamente predispuestos a padecerla $\left({ }^{2}\right)$.

El incremento del síndrome metabólico va asociado a la expansión de la epidemia mundial de diabetes tipo 2 y de enfermedades cardiovasculares, según datos recientes de la Federación Internacional de Diabetes (FID). Las personas con síndrome metabólico -20 a $25 \%$ de la población mundial $\left({ }^{3}\right)$ - tienen una probabilidad tres veces mayor de sufrir un ataque cardíaco o un accidente cerebro vascular y dos veces más de morir por estas causas, que las personas que no lo padecen $\left({ }^{4}\right)$.

Lamentablemente, existen diferentes criterios para identificar a las personas con síndrome metabólico. Aquellos que promueven cada uno de ellos, dan sus propios fundamentos a favor del uso del que recomiendan. En el presente estudio, se usará el recomendado por el National Cholesterol Education Program ATP III (Adult Treatment Panel) $\left({ }^{5}\right)$, dado que es el más práctico para estudios epidemiológicos.

En el Perú, utilizando este criterio, existen varios estudios: en el departamento de Lambayeque, en un estudio de representación departamental, se encontró $28,3 \%$ de síndrome metabólico en mayores de 30 años de edad, 29,9\% en el género femenino y en el masculino $23,1 \%$, siendo esta diferencia estadísticamente significativa $\left(^{6}\right)$. En el mismo departamento, comparando un grupo de pescadores contra uno de agricultores y de edades comprendidas entre los 30 y 70 años, se encontró 31,7 y $22,1 \%$, respectivamente. Acá también se encontró que el género femenino presentaba una mayor prevalencia $(31,8 \%)$ que el masculino $(20,8 \%)$. La prevalencia del síndrome metabólico en toda la población fue $26,7 \%\left({ }^{7}\right)$.

En Lima Metropolitana, en una población urbana, de 30 a 92 años, se encontró $14,4 \%$, el $16,3 \%$ en el género femenino y $10 \%$ en el masculino $\left({ }^{8}\right)$. En un estudio realizado en mujeres adultas con sobrepeso y obesidad, de Lima Metropolitana, se comunicó una prevalencia de síndrome metabólico de 28 y $30 \%$, respectivamente $\left({ }^{9}\right)$. Una tesis realizada en Cusco, en mujeres posmenopáusicas, hallaron $16,5 \%$ con síndrome metabólico $\left({ }^{10}\right)$. En el mismo departamento, cuando se comparó un distrito urbano, Wanchaq, frente a uno rural, Huancarani, se encontró 6 y $0,8 \%$, respectivamente $\left({ }^{11}\right)$.

El objetivo del presente estudio fue conocer la prevalencia del síndrome metabólico en la población adulta peruana. Los estudios locales mencionados ya mostraban una presencia importante de este síndrome y era necesario identificar lo que sucedía en el ámbito nacional.

\section{MATERIALES Y MÉTODOS}

La información ha sido tomada de la Encuesta Nacional de Indicadores Nutricionales, Bioquímicos, Socioeconómicos y Culturales relacionados con las enfermedades crónicas degenerativas, realizada por la Dirección de Vigilancia Alimentario Nutricional del Centro Nacional de Alimentación y Nutrición ${ }^{(2)}$.

Se realizó un muestreo, por conglomerado estratificado. Se consideró 5 estratos geográficos, que han sido determinados para este estudio: Lima Metropolitana, resto de la costa, sierra urbana, sierra rural y selva. En cada estrato, se seleccionó los conglo- 
merados poblacionales definidos por el Instituto Nacional de Estadística e Informática. Esta selección se realizó por muestreo simple aleatorio sin reemplazo. Dentro de cada conglomerado, se aplicó la metodología de selección estandarizado para seleccionar manzanas, viviendas y personas. De esta forma, el diseño muestral tuvo cuatro etapas de selección. Para conocer en detalle el tipo de muestreo y todo lo concerniente a él, se remite al lector al trabajo original $\left({ }^{12}\right)$.

Se estudió 4091 personas mayores de 20 años, de ambos géneros; $50,4 \%$ correspondió al género femenino y $49,6 \%$, al masculino.

Los resultados se presenta en forma nacional y por los siguientes ámbitos: resto de la costa, sierra urbana, sierra rural, selva y Lima Metropolitana.

Los indicadores antropométricos fueron peso, talla y circunferencia de la cintura (CC). Con los dos primeros, se calculó el índice de masa corporal (peso/talla ${ }^{2}$, expresado en $\mathrm{kg} / \mathrm{m}^{2}$. Todas las medidas fueron tomadas siguiendo las recomendaciones internacionalmente aceptadas $\left({ }^{13,14}\right)$.

Como indicadores bioquímicos, se dosó glicemia y perfil lipídico (colesterol total, C-HDL, C-LDL, C-VLDL y triglicéridos). Por las características del trabajo, solo se tomó en cuenta los valores del C-HDL y los triglicéridos.

La presión arterial fue tomada de acuerdo a la metodología conocida.

Se hizo el diagnóstico de síndrome metabólico cuando estuvieron presentes 3 o más de los factores de riesgo, de acuerdo a los criterios del ATP III $\left.{ }^{5}\right)$. (Tabla 1 )

\section{RESULTADOS}

La prevalencia nacional del síndrome metabólico fue $16,8 \%$. Este porcentaje na-
Tabla 1. Factores de riesgo que estuvieron presentes, de acuerdo a los criterios de ATP III.

\begin{tabular}{ll}
\hline \multicolumn{1}{c}{ Factor de riesgo } & \multicolumn{1}{c}{ Definición } \\
\hline Obesidad abdominal & CC $\geq 102 \mathrm{~cm}$ en hombres y \\
& $\geq 88 \mathrm{~cm}$ en mujeres \\
& $\geq 150 \mathrm{mg} / \mathrm{dL}$ \\
Triglicéridos & $<40 \mathrm{mg} / \mathrm{dL}$ en hombres y \\
Colesterol HDL & $<50 \mathrm{mg} / \mathrm{dL}$ en mujeres \\
& $\geq 130 / \geq 85 \mathrm{mmHg}$ \\
Presión arterial & $\geq 110 \mathrm{mg} / \mathrm{dL}$ \\
Glucosa &
\end{tabular}

CC: Circunferencia de la cintura.

cional solo fue superado por Lima Metropolitana $(20,7 \%)$ y el resto de la costa $(21,5 \%)$. La menor prevalencia se dio en la sierra rural, con $11,1 \%$. Los datos sugieren que en aquellos ámbitos donde está menos presente la transición epidemiológica se encuentran menos expuestos a las alteraciones en sus indicadores (Figura 1).

En cuanto a lo que concierne al género, indudablemente el femenino es el más afectado; la presencia del síndrome metabólico fue $26,4 \%$, lo que significa que, aproximadamente, una de cada cuatro mujeres en el Perú ya lo presentaba. Con relación al género masculino, solo lo tuvo $7,2 \%$. Según los ámbitos, Lima Metropolitana y

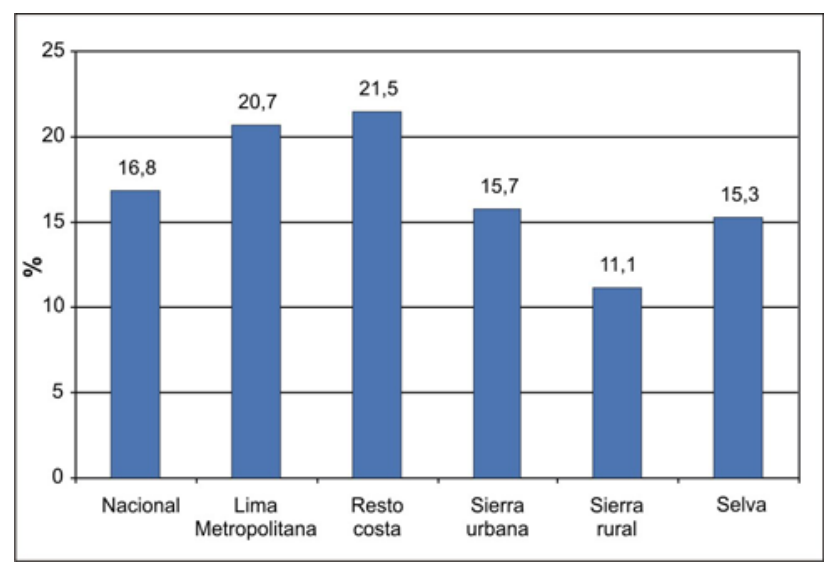

Figura 1. Prevalencia de síndrome metabólico, según factores de riesgo. Criterios ATP III. 


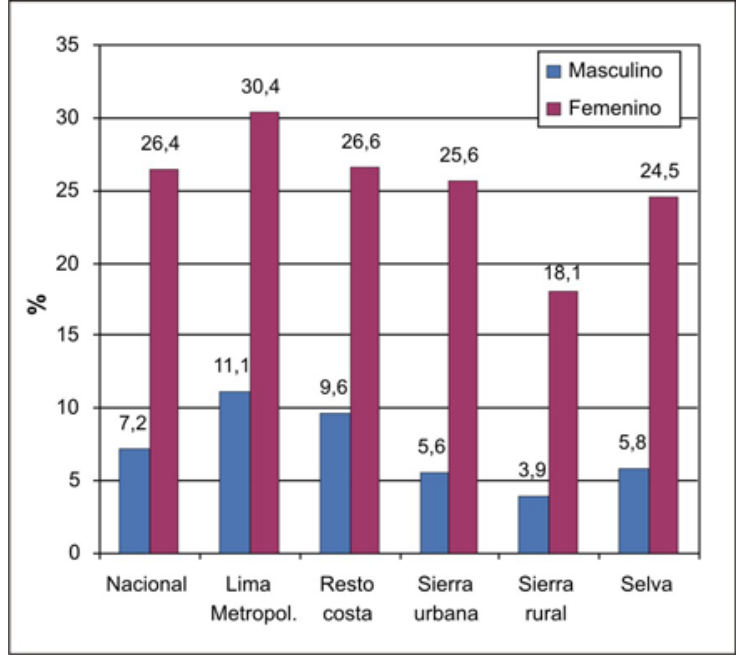

Figura 2. Prevalencia de síndrome metabólico, según género.

el resto de la costa fueron los que tuvieron mayor prevalencia para ambos géneros (Figura 2).

Si se relaciona el síndrome metabólico con el estado nutricional dado por el IMC (normal, sobrepeso y obesidad), se observa que la presencia del síndrome metabólico tiene un comportamiento lineal: a mayor IMC más síndrome metabólico. La diferen-

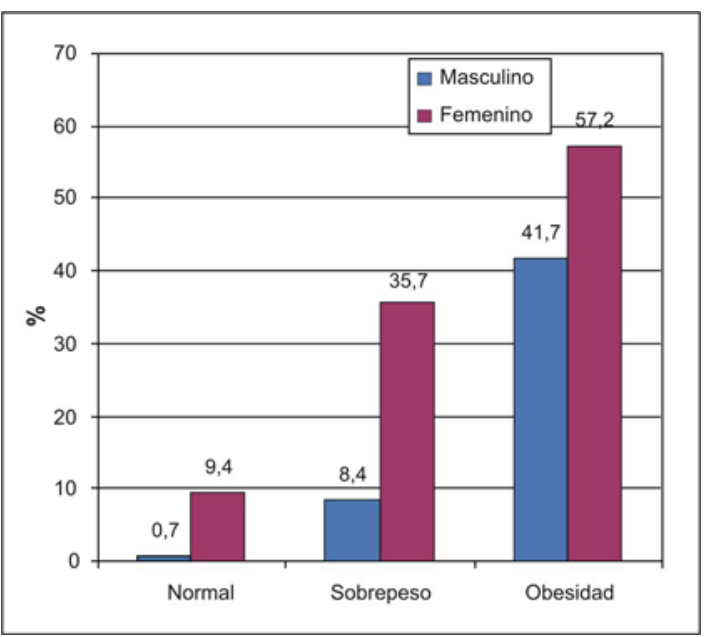

Figura 3. Prevalencia de síndrome metabólico de acuerdo a su estado nutricional, según género. cia de género también se dio como el gráfico anterior. El Figura 3 muestra muy claramente los valores altos de síndrome metabólico en las personas con sobrepeso y mucho mayor en los con obesidad; en el género femenino, una de cada dos obesas tenía el síndrome metabólico. Lo interesante fue la presencia de síndrome metabólico en personas con un IMC considerado normal.

Dentro de los 5 factores de riesgo utilizados para el diagnóstico de síndrome metabólico, primó la mayor cantidad de personas del género femenino que tuvieron la circunferencia de la cintura $(50,9 \%)$ y el CHDL $(86,8 \%)$ en niveles superiores a lo estipulado en el ATP III, con relación a lo encontrado en el género masculino $(10,7$ y $25,6 \%$, respectivamente). Lo contrario sucedió para la presión arterial. Donde prácticamente no existieron diferencias fue en los triglicéridos y en la glicemia (Figura 4).

Las categorías de riesgo dadas por la circunferencia de la cintura están determinadas por los siguientes valores: categoría 0 sin riesgo $(\mathrm{CC}<80$ y $<94 \mathrm{~cm})$; categoría 1 de riesgo $(\mathrm{CC}>80 \mathrm{y} \leq 88,>94 \mathrm{y} \leq$ $102 \mathrm{~cm})$ y categoría 2 de mucho riesgo ( $>$

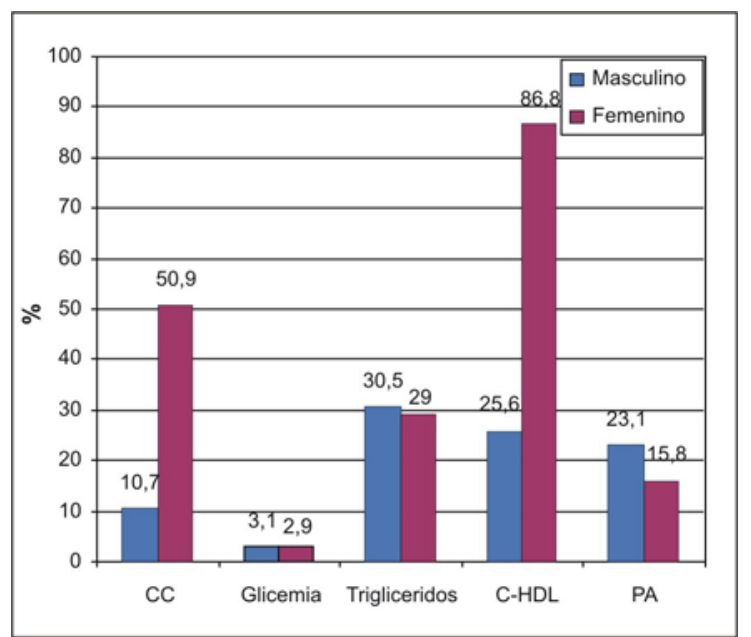

Figura 4. Porcentaje de personas de acuerdo a indicadores de riesgo, según género. 
Jaime Pajuelo y col.

Tabla 2. Promedio y desvío estándar de las variables que componen los criterios del síndrome metabólico, de acuerdo a valores de circunferencia de la cintura (CC), según género.

\begin{tabular}{|c|c|c|c|c|c|c|}
\hline & \multicolumn{3}{|c|}{ Masculino } & \multicolumn{3}{|c|}{ Femenino } \\
\hline & 0 & 1 & 2 & 0 & 1 & 2 \\
\hline $\mathrm{CC}(\mathrm{cm})$ & $83,3(6,4)$ & $97,5(2,2)$ & $108,7(7,2)$ & $74,1(4,5)$ & $83,9(2,4)$ & $98,1(8,1)$ \\
\hline Glicemia (mg/dL) & $80,8(21,8)$ & $87,1(19,2)$ & $92,7(27,6)$ & $78,3(16,4)$ & $80,1(20)$ & $87,3(27,3)$ \\
\hline Triglicéridos (mg/dL) & $117,8(5,6)$ & $172,5(77,9)$ & $192,7(80,6)$ & $102(44,4)$ & $116,9(56,5)$ & $150,6(73,1)$ \\
\hline $\mathrm{C}-\mathrm{HDL}$ (mg/dL) & $43,7(5,8)$ & $42,1(4,4)$ & $41,6(3,4)$ & $44,7(6,5)$ & $43,9(5,6)$ & $43,2(5)$ \\
\hline Presión sistólica (mmHg) & $111(15,2)$ & $119,6(17,5)$ & $126,3(18,5)$ & $103(14,1)$ & $106,3(15,8)$ & $113,8(18,6)$ \\
\hline Presión diastólica (mm/Hg) & $69,5(11)$ & $73,2(11,6)$ & $77,6(12,3)$ & $64,6(10)$ & $67(11,1)$ & $70,7(11,5)$ \\
\hline
\end{tabular}

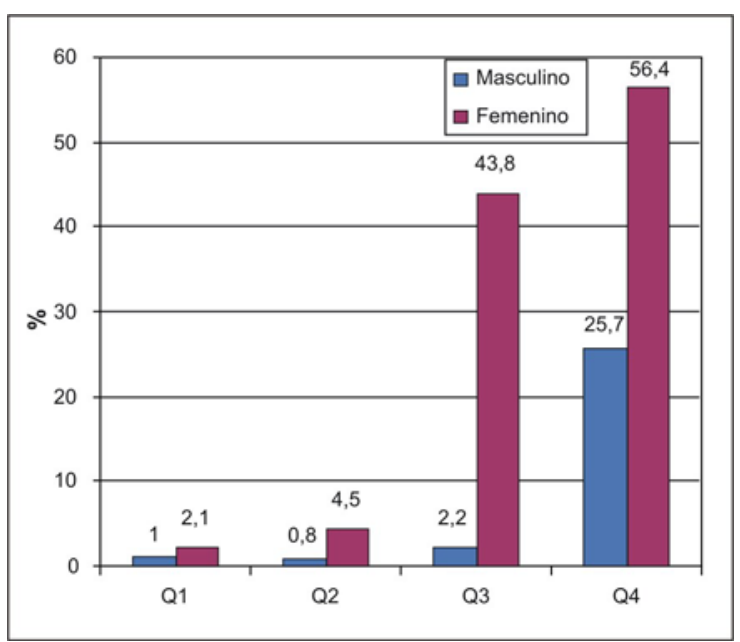

Figura 5. Prevalencia de síndrome metabólico de acuerdo a cuartiles (Q) de circunferencia de cintura, según género.

88 y > $102 \mathrm{~cm}$ ), para los géneros femenino y masculino, respectivamente. Lo que muestra la Tabla 2 es que, conforme se incrementa la circunferencia de la cintura, la presión diastólica y presión sistólica, conjuntamente con las otras variables bioquímicas que se usa para identificar el síndrome metabólico, tienen una respuesta similar. Todas las variables presentaron significación estadística $(p<0,001)$. La única que tuvo un comportamiento diferente fue la HDL, donde no se encontró diferencia estadísticamente significativa, entre la categoría 1 y 2.
En el Figura 5 se muestra la presencia del síndrome metabólico de acuerdo a cuartiles (Q). La poca presencia de síndrome metabólico en los Q1 y Q2 se debe a trastornos bioquímicos y/o a la presencia de presión arterial, pero ya en los Q3 y Q4 se suma una circunferencia de la cintura incrementada. Lo interesante a destacar es que el género femenino fue el más afectado.

La presencia del síndrome metabólico de acuerdo a la edad tendió a incrementarse. A mayor edad, mayor prevalencia; y eso sucedió en ambos géneros. Este gráfico también muestra que el género más afectado fue el femenino (Figura 6).

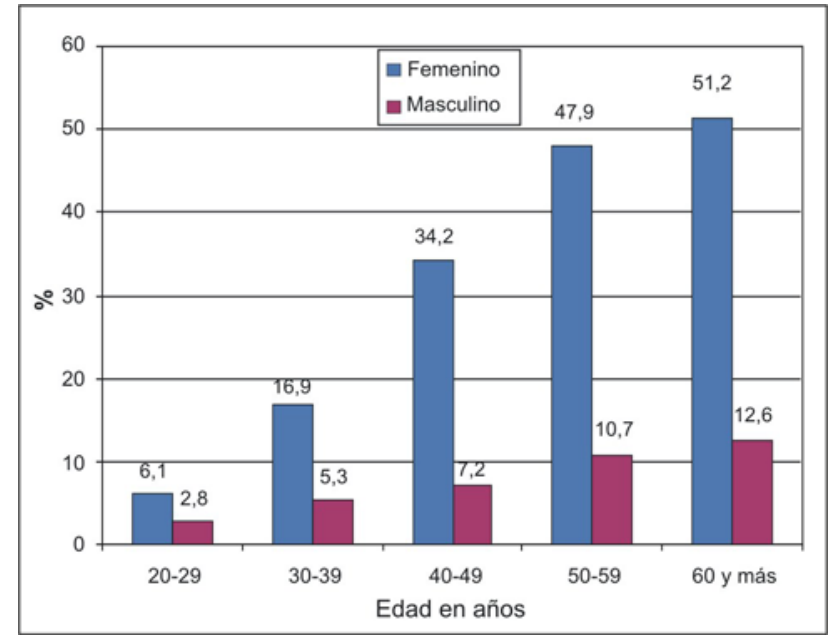

Figura 6. Prevalencia de síndrome metabólico por grupos de edad, según género. 


\section{DISCUSIÓN}

El conocimiento de la situación actual en el Perú, con relación a las enfermedades crónicas no transmisibles, ha sido gracias a un estudio realizado por el Centro Nacional de Alimentación y Nutrición. El mencionado estudio ha comunicado la siguiente información: la prevalencia de sobrepeso y obesidad alcanza el $51,8 \%$, la hipercolesterolemia $19,6 \%$, la hipertrigliceridemia $15,3 \%$, la disminución del C-HDL $1,1 \%$, el incremento de la LDL $15,3 \%$, la hipertensión arterial $13,3 \%$ y la diabetes mellitus, $2,8 \%\left({ }^{12}\right)$. Todas estas patologías son consideradas de riesgo cardiovascular.

La mencionada información proporciona una visión de magnitud de cada patología, en forma independiente de las otras, mientras que el síndrome metabólico, eligiendo alguna de ellas e incorporando a otras, da un panorama de conjunto, ya que asocia una serie de variables en un mismo individuo. Sin embargo, existe más de una forma de agrupar las variables que constituyen el síndrome metabólico. Cada autor da sus fundamentos para ser usados. El síndrome metabólico es un método simple que tiene similar capacidad para predecir incidentes cardiovasculares y diabetes mellitus, aunque ellos tienen diferente sensibilidad $y$ especificidad $\left({ }^{15}\right)$.

Para este trabajo, se ha elegido el criterio del ATP III, que es el que mejor se acomoda para estudios epidemiológicos; e incluso para los clínicos, ya que estos prefieren un instrumento simple que les permita evaluar a pacientes y realizar mejor su tratamiento $\left({ }^{16}\right)$.

De acuerdo a los resultados hallados se puede afirmar que 2680000 personas adultas en el país presentaban el síndrome metabólico, estando mayormente localizadas en Lima Metropolitana y el resto de la costa; y esto se explicaría por cuanto en estas áreas es donde más se puede observar el fenómeno de la transición epidemiológica. En países como Estados Unidos, donde la presencia de la transición es mucho más homogénea, aproximadamente, un tercio de personas presenta el síndrome metabólico $\left({ }^{17}\right)$.

La presencia del síndrome metabólico en mayor medida en el género femenino se debería, primero a que el sobrepeso y la obesidad es más prevalerte y segundo a que 2 de los criterios para definir el síndrome metabólico (circunferencia de la cintura y C-HDL) son marcadamente diferentes en este género. En China, encontraron una prevalencia de síndrome metabólico de $9,8 \%$ en el género masculino y $17,8 \%$ en el femenino $\left({ }^{18}\right)$. Cifras mas bajas que las nuestras.

En el presente trabajo, se observa nítidamente que el síndrome metabólico se encuentra más en las personas con sobrepeso y mucho más en los obesos. Esta tendencia también ha sido encontrada por Park, quien halló 5\% de síndrome metabólico en las personas normales, $22 \%$ en aquellas con sobrepeso y $60 \%$ en las obesas $\left({ }^{19}\right)$.

Una de las opiniones controversiales en el uso del ATP III, se refiere a los valores de circunferencia de la cintura para identificar riesgo, por cuanto se menciona que nuestra población es más parecida a la de países asiáticos y que por ende no debería usarse los valores que da el ATP, sino que tendrían que ser menores. Un informe de la oficina regional del Pacífico Occidental de la Organización Mundial de la Salud, la International Association for the Study of Obesity y la International Obesity Task Force recomiendan que los puntos de corte de la circunferencia de la cintura deberían ser 80 y $90 \mathrm{~cm}$ para mujeres y hombres, respectivamente, para la población asiática $\left({ }^{20}\right)$.

En este sentido, la pregunta que cabe hacerse es a qué países asiáticos se refiere, por cuanto en el Asia así como en nuestra 
América Latina no somos todos iguales. En China, por ejemplo, la mayor prevalencia de obesos (IMC > $>25 \mathrm{~kg} / \mathrm{m}^{2}$ ) llega a $31 \%$, mientras que en nuestro país es de $51 \%\left({ }^{18}\right)$.

La obesidad abdominal es la manifestación más prevalerte del síndrome metabólico, es un marcador de disfunción del tejido adiposo y esto es de importancia central en el diagnóstico clínico ${ }^{(21)}$. La circunferencia de la cintura es un indicador antropométrico y como tal no escapa a que sus niveles diagnósticos sean elegidos arbitrariamente y que esa elección responda más a criterios estadísticos que biológicos.

Independientemente del nivel que se tome, se debe reconocer que la circunferencia de la cintura es un gran indicador de riesgo, dado que se ha visto que conforme esta se incrementa, la glucosa, los triglicéridos y la presión arterial sistólica y presión arterial diastólica hacen lo mismo, mientras que el colesterol HDL disminuye; aunque, esto último no presenta significación estadística. En la población asiática, también se muestra que el incremento de la circunferencia de la cintura conlleva un incremento en la presión arterial, triglicéridos y glucosa $\left({ }^{22}\right)$.

Por otro lado, cuando se divide el universo en cuatro partes iguales (Q), se observa nítidamente la asociación que existe con el síndrome metabólico. Sin embargo, es necesario comentar que en las personas con síndrome metabólico que corresponde a los dos primeros cuartiles (Q1 y Q2), no está presente la circunferencia de la cintura como criterio diagnóstico.

En un estudio realizado en Singapore, se demostró que tanto en el grupo de individuos con síndrome metabólico, donde se incluía la $\mathrm{CC}$, como en otro grupo con síndrome metabólico pero sin la presencia de la $\mathrm{CC}$, los riesgos de la enfermedad cardiaca isquémica eran iguales $\left({ }^{23}\right)$.

Usando el ATP III con relación al Federación Internacional de Diabetes (FID) y con un período de observación de 10 años, el $10,7 \%$ de aquellos diagnosticados con síndrome metabólico con el ATP hicieron un evento cardiovascular, frente a $5,5 \%$ de los diagnosticados con el FID. Los autores concluyen que, pese a que la prevalencia de síndrome metabólico fue más alta con los criterios del FID, este tiene poder predictivo bajo para eventos coronarios $\left({ }^{24}\right)$.

Hasta tanto existan evidencias que permitan modificar estos valores de corte, considero que debe seguirse utilizando lo que señala el ATP, dado que así se puede llegar a comparar trabajos, además que son indicadores que pueden ser utilizados masivamente.

El manejo del síndrome metabólico es atacar los factores que lo promueven, como son el sobrepeso y la obesidad, resultante de la inactividad física y cambio en los patrones dietarios $\left({ }^{25}\right)$.

El IMC, si bien no está dentro de los criterios diagnósticos usados para el síndrome metabólico, es muy importante, por cuanto las alteraciones tanto bioquímicas como de la presión arterial guardan cierta relación lineal. Tal es así que, si se observa las prevalencias del síndrome metabólico por ámbito y las de sobrepeso y obesidad, serían explicadas de alguna manera. El sobrepeso y la obesidad para Lima Metropolitana fue $59,7 \%$, resto de la costa $56,4 \%$, sierra urbana $45,3 \%$, sierra rural $28,8 \%$ y selva $43,4 \%$.

Independientemente de la discusión que se tiene sobre cuál de los criterios para definir el síndrome metabólico se debe utilizar, no se debe perder de vista que el principal problema que se tiene es el sobrepeso y la obesidad y que estos, de alguna manera, generan una serie de trastornos metabólicos que van a conllevar a incrementos de los diferentes riesgos que se conoce. Por esa razón es que, las principales acciones deben estar dirigidas en el campo 
de la prevención y por último en la terapéutica. El objetivo es reducir de peso y mantener esa pérdida. Y esto se alcanza con una reducción en la ingesta calórica y un régimen de ejercicio. Existe una serie de trabajos que así lo demuestran $\left({ }^{26-28}\right)$.

Los responsables de velar por la salud de nuestra población deben tomar cartas en el asunto, dado que este tipo de expresiones de 'anormalidad' tiene una tendencia epidemiológica a continuar incrementándose, acarreando problemas no solo para la salud sino para la economía del país. Otro hecho a tomar en cuenta, dentro de las características epidemiológicas del síndrome metabólico es que, conforme avanza la edad la prevalencia aumenta y como la expectativa de vida al nacer ha mejorado, se van a presentar más los problemas.

\section{REFERENCIAS BIBLIOGRÁFICAS}

1. George K, Alberti M, Zimmet P, Shaw J. The metabolic syndrome-a new worldwide definition. The Lancet. 2005;366:1059-62.

2. Groop L, Orho-Melander M. The dysmetabolic syndrome. J Intern Med. 2001;250:105-20.

3. Dunstan D, Zimmet P, Welborn T, De Courten M, Cameron A, Sucre R, et al. The rising prevalence of diabetes and impaired glucosa tolerante. Diabetes Care. 2002;25:82934.

4. Isomaa B, Almeren P, Tuomi T, Forsen B, Lahti K, Nissen $\mathrm{M}$, et al. Cardiovascular morbidity and mortality associated with the metabolic syndrome. Diabetes Care, 2001;24(4):6839.

5. Expert Panel on Detection, Evaluation and Treatment of High Blood Cholesterol in Adults. Executive summary of the Third Report of the National Cholesterol Education Program (NCEP) Expert Panel Detection, Evaluation and Treatment of High Blood Cholesterol in Adults (Adult Treatment Panel III). JAMA. 2001;285:2486-97.

6. Soto V, Vergara E, Neciosup E. Prevalencia y factores de riesgo de síndrome metabólico en población adulta de Lambayeque. Rev Peru Med Exp Salud Pública. 2005:22(4):254-61.

7. Guarnizo M, Loayza G, Calvay M, Inami M, Lázaro H. Síndrome metabólico en una población pesquera y otra agropecuaria de la Costa del Perú. Rev Soc Peru Med Interna. 2006;19:10-8.

8. Seclen S, Villena A, Serrano Rios M, Gamarra D, Pinto M. Prevalencia del Síndrome Metabólico en una población urbana de Lima. XV Jornadas Científicas de la Universidad Particular Cayetano Heredia. Lima: UPCH; 2002.

9. Pajuelo J. El Síndrome Metabólico en mujeres adultas según el estado nutricional. X Congreso Peruano de Endocrinología. Lima: Sociedad Peruana de Endocrinología; 2006.

10. Mena I. Influencia de la edad y del uso de terapia de reemplazo hormonal en la prevalencia del Síndrome Metabólico en mujeres post menopáusicas. Tesis para optar el Grado Académico de Médico Cirujano. Arequipa: Universidad Nacional de San Agustin, Facultad de Medicina; 2005.

11. Gamarra M. Prevalencia comparativa de Síndrome Metabólico entre un distrito urbano y uno rural del Departamento del Cusco. X Congreso Peruano de Endocrinología. Lima: Sociedad Peruana de Endocrinología; 2006.

12. Ministerio de Salud. Instituto Nacional de Salud (INS). Centro Nacional de Alimentación y Nutrición (CENAN). Encuesta Nacional de Indicadores Nutricionales, Bioquímicos, Socioeconómicos y Culturales relacionados con las enfermedades crónicas degenerativas. Lima: INS-CENAN; 2006.

13. Lohman T, Roche A. Anthropometric Standardization Referent Manual. Illinois, Champaign: Human Kinetics Books; 1990.

14. Lean M, Han T, Deurenberg P. Predicting body composition by densitometry from simple anthropometric measurements. Am J Clin Nutr. 1996;63:4-14.

15. Lorenzo C, Williams K, Hunt K, Haffner S. The National Cholesterol Education Program-Adult Treatment Panel III, International Diabetes Federation, and World Health Organization Definitions of the Metabolic Syndrome as Predictors of Incident Cardiovascular Disease and Diabetes. Diabetes Care. 2007;30:8-13.

16. Ecket R, Grundy S, Zimmet P. The metabolic syndrome. The Lancet. 2005;365:1415-28.

17. Ford E, Giles W, Dietz W. Prevalence of the metabolic syndrome among US adults: findings from the third National Health and Nutrition Examination Survey. JAMA. 2002;287:356-9.

18. Gu D, Reynold K, Wu X, Chen J, Duan X, Reynolds R, et al. For the InterASIA Collaborative Group. Prevalence of the metabolic syndrome and overweight among adults in China. The Lancet. 2005;365:1398-1405.

19. Park Y, Zim S, Palaniappan L, Heshka S, Carnethon M, Heymsfield $S$, et al. The metabolic syndrome: Prevalence and associated risk factors findings in the US population from the Third National Health an Nutrition Examination Survey. Arch Inter Med. 2003;163:427-36.

20. The World Health Organization Western Pacific Region, the International Association for the Study of Obesity, and the International Obesity Task Force. The Asia Pacific perspective redefining obesity and its treatment. Melbourne: Health Communications, Australia; 2000.

21. Després JP, Lemieux I. Abdominal obesity and metabolic syndrome. Nature. 2006;444:881-2.

22. Wildman RP, Gu D, Reynolds K, Duan X, Wu X, He J. Are waist circumference and body mass index independently 
associated with cardiovascular disease risk in Chinese adults? Am J Clin Nutr. 2005;82:1195-9.

23. Lee J, Ma S, Heng D, Tan Ch, Chef S, Hughes K, et al. Should central obesity be an optional or essential component of the metabolic syndrome? Ischemic heart disease risk in the Singapore Cardiovascular Cohort Study. Diabetes Care. 2007;30:343-7.

24. Assmann G, Guerra R, Fox G, Cullen P, Schulte H, Willett $\mathrm{D}$, et al. Harmonizing the definition of the metabolic syndrome: comparison of the criteria of the Adult Treatment Panel III and the International Diabetes Federation in United States American and European populations. Am J Cardiol. 2007;99:541-4.

25. Grundy SM, Hansen B, Smith SC Jr, Cleeman JI, Kahn RA; American Heart Association; et al. Clinical management of metabolic syndrome: report of the American Heart Association/National Heart, Lung, and Blood Institute/ American Diabetes Association conference on scientific issues related to management. Arterioscler Thromb Vasc Biol. 2004;24(2):e19-24.

26. Meydani M. A Mediterranean-style diet and metabolic syndrome. Nutr Rev. 2005;63:312-4.
27. Esposito K, Marfella R, Ciotola M, Di Palo C, Giugliano F, Giugliano G, et al. Effect of a Mediterranean-style diet on endothelial dysfunction and markers of vascular inflammation in the metabolic syndrome: a randomized trial. JAMA. 2004;292:1440-6.

28. Clinical Guidelines on the Identification, Evaluation, and Treatment of Overweight and Obesity in Adults-The Evidence Report. National Institutes of Health. Obes Res. 1998;6 Suppl 2:51S-209S.

Manuscrito recibido el 26 de febrero de 2007 y aceptado para publicación el 23 de marzo de 2007.

\section{Correspondencia:}

Jaime Pajuelo Ramírez

Instituto de Investigaciones Clínicas

Hospital Nacional Dos de Mayo.

Av. Grau cuadra 13. Parque Historia de la Medicina.

Lima 1, Perú.

Correo-e: saturse@speedy.com.pe 